\title{
Reverse Link Performance of DS-CDMA Cellular Systems through Closed-Loop Power Control and Beamforming in 2D Urban Environment
}

\author{
Mohamad Dosaranian-Moghadam ${ }^{1}$, Hamidreza Bakhshi², and Gholamreza \\ Dadashzadeh $^{2}$ \\ ${ }^{1}$ Department of Electrical Engineering, Islamic Azad University, Qazvin Branch, \\ Qazvin, Iran \\ m_dmoghadameqiau.ac.ir \\ ${ }^{2}$ Department of Electrical Engineering, Shahed University, Tehran, Iran \\ bakhshieshahed.ac.ir \\ gdadashzadeheshahed.ac.ir
}

\begin{abstract}
The interference reduction capability of antenna arrays and the power control algorithms have been considered separately as means to decrease the interference in wireless communication networks. In this paper, we propose smart step closed-loop power control (SSPC) algorithm in direct sequence-code division multiple access (DS-CDMA) receivers in a $2 D$ urban environment. This RAKE receiver consists of conjugate gradient adaptive beamforming $(C G B F)$ and matched filter $(M F)$ in two stages and finally, the output signals from the MFs are combined and then are fed into the decision circuit for the desired user. Also, we present switched-beam (SB) technique for enhancing signal to interference plus noise ratio (SINR) in network. Also, we study an analytical approach for the evaluation of the impact of power control error (PCE) on DS-CDMA systems in a $2 D$ urban environment. The simulation results indicate that the convergence speed of the SSPC algorithm is faster than other algorithms. Also, we observe that significant saving in total transmit power (TTP) are possible with our proposed algorithm. Finally, we discuss two parameters of the PCE and path-loss exponent and their effects on capacity of the system via some computer.
\end{abstract}

\section{KEYWORDS}

Closed-loop power control, conjugate gradient adaptive beamforming, DS-CDMA, power control error, matched filter

\section{INTRODUCTION}

Code-division multiple access (CDMA) for cellular communication networks requires the implementation of some forms of adaptive power control. In the uplink of CDMA systems, the maximum number of supportable users per cell is limited by multipath fading, shadowing, and near-far effects that cause fluctuations of the received power at the base station (BS). Two types of power control are often considered: closed-loop power control and open-loop power control [1], [2]. In a closed-loop power control, according to the received signal power at a base station, the base station sends a command to a mobile set (MS) to adjust the transmit power of the mobile. Also, closed-loop power control is employed to combat fast channel fluctuations due to fading. Closed-loop algorithms can effectively compensate fading variations when the power control updating time is smaller than the correlation time of the channel. However, in an openloop power control, a mobile set adjusts its transmit power according to its received power in the downlink [1]-[6]. Accordingly, in this paper we present smart step closed-loop power control (SSPC) algorithm for minimizing the total transmit power (TTP) in DS-CDMA cellular systems [7]-[10].

DOI : $10.5121 /$ ijenc. 2010.2610 
Diversity and power control are two effective techniques for enhancing the signal to interference plus noise ratio (SINR) for wireless networks. Diversity exploits the random nature of radio propagation by finding independent (or, at least, highly uncorrelated) signal paths for communication. If one radio path undergoes a deep fade, another independent path may have a strong signal. By having more than one path to select from, the SINR at the receiver can be improved. The diversity scheme can be divided into three methods: 1) the space diversity; 2) the time diversity; 3) the frequency diversity. In these schemes, the same information is first received (or transmitted) at different locations (or time slots/frequency bands). After that, these signals are combined to increase the received SINR. The antenna array is an example of the space diversity, which uses a beamformer to increase the SINR for a particular direction [6]. Accordingly, the use of smart antennas is expected to have a significant impact on future wireless communications to meet the projected perspective of future communication networks. A major reason to use smart antenna in wireless communication is its capability to intelligently respond to the unknown interference environment in real time. The process of formation of nulls in the direction of interference and strong beams in the direction of desired user is called adaptive array processing. These systems are called adaptive beamforming system and consist of spatially disposed sensor elements connected to a single channel or to a multi channel adaptive processor. The term adaptive beamforming is also referred as smart antennas. Adaptive antenna array can be used to eliminate the directional interference by adaptive canceling and therefore to improve the SINR. Steering capability of adaptive array depends on processing algorithms for null steering. Such algorithms are called adaptive algorithm. In wireless communications, smart antennas are used due to their ability to separate the desired signal from interfering signals. By knowing the direction of the desired signals, they are able to adjust the antenna pattern intelligently by adjusting the weights of the adaptive algorithm [1]-[3].

The goal of this paper is to extend the works in [7]-[17] by considering joint multiple-cell system, adaptive beamforming, closed-loop power control, and power control error (PCE) in a 2D urban environment. In [7]-[10], we proposed the SSPC algorithm in DS-CDMA cellular systems in multipath fading channels without considering the PCE. In [11], we considered the joint SSPC algorithm, adaptive beamforming, and PCE over multipath fading. In [12], we considered DS-CDMA systems performance with base station assignment, PCE, and beamforming in the presence of frequency-selective Rayleigh fading channel. In [13] and [14], we considered the joint SSPC algorithm and constrained least mean squared (CLMS) algorithm for wireless networks in a 2D urban environment. Also in [15], we presented switched-beam (SB) technique in CDMA cellular systems in a 2D urban environment with centralized power control. Also in [16] and [17], a RAKE receiver in a single-cell system was proposed in the presence of frequency-selective Rayleigh fading channel. Accordingly, in this paper we present the SSPC algorithm in DS-CDMA cellular systems in a 2D urban environment. On the other hand, the issue of the effect of power control errors on wireless networks has received a great deal of attention over the last few years [1], [2], [18]. Finally, we consider the effect of PCE on DS-CDMA cellular systems in a 2D urban environment.

In this paper, a RAKE receiver in DS-CDMA system is analyzed in two stages according to Figure 1 [16]. In the first stage, this receiver uses conjugate gradient (CG) adaptive beamforming to find optimum antenna weights assuming perfect estimation of the channel parameters (direction, delay, and power) for the desired user. The desired user resolvable paths' directions are fed to the CG beamformer to cancel out the inter-path interference (IPI) from other directions. Also, the RAKE receiver uses conventional demodulation in the second stage to reduce multiple-access interference (MAI). Reducing the MAI will further decrease the system BER.

The organization of the remainder of this paper is as follows. In Section 2, propagation model in a 2D urban environment and also functional state of urban signal propagation simulator (USPS) are described. The system model is given in Section 3. The RAKE receiver structure is 


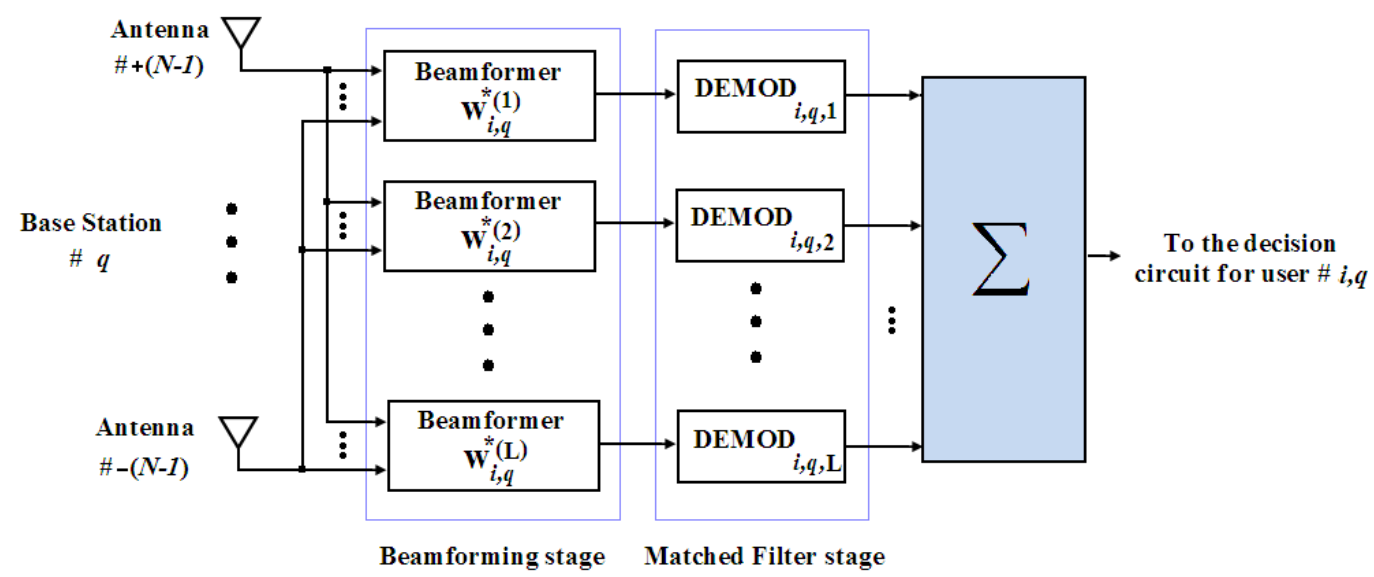

Figure 1. Block diagram of a two-stage RAKE receiver in DS-CDMA system [16]

described in Section 4. In Section 5, we propose the SSPC algorithm. Then in Section 6, we extend the analysis to the case of PCE on DS-CDMA cellular systems in a 2D urban environment. Section 7 describes the SB technique. Finally, simulation results and conclusions are given in Sections 8 and 9, respectively.

\section{Propagation Model In 2D Urban Environment}

Because of using 2D urban structure in this paper, for computing yield for path between a mobile set and base station, propagation model in urban environments are dramatized. In a propagation model of urban environments and in reverse link (uplink), mobile set antenna is radiating beams which are diffusing in all directions and parts of beams reach to base station.

In urban environment, delivered beam from mobile set by the time of collision to an obstacle like a wall surface or a building, reflects to a new angle and continues its path, this is called reflection phenomena. In condition that radiated beam is conflicted to an obstacle edge, then diffraction phenomena is happened and diffracting point is diffusing new beams to all directions like a transmitter. All reflected beams, will stay in the environment till the time their power are not reduced to a threshold limit. Figure 2 shows both phenomena in reverse link and for line of sight (LoS) and non-LoS paths [15], [19].

According to above dramatization, we could see because of LoS in un-urban environment, only one signal is delivered from each user to receiver, while in function and because of elimination phenomena in an urban environment, beside to signals which are delivered to line sight, signals which have difference in phase or domain with this signal are also received by receiver. In this paper, the software USPS is used to implement a 2D urban environment.

\section{SYSTEM MODEL}

In this paper, we focus on the uplink communication paths in a DS-CDMA cellular system in a 2D urban environment. The channel is modeled by software USPS according to section 2 with lognormal distributed shadowing. Initially, we consider $L_{k}$ paths for each link for user $k$ that optimally combined through a RAKE receiver according to Figure 1 that for simplicity, we assume $L=\min _{k}\left(L_{k}\right)$ for all users. Also, we assume that there are $M$ active base stations in the network, with $K_{m}$ users connected to $m$ th base station. At each base station, an antenna array of $S$ sensors and $N$ weights is employed, where $S=2 N-1$, to receive signals from all users. Note that in CG adaptation algorithm, unlike other adaptation algorithms, the number of weights is 


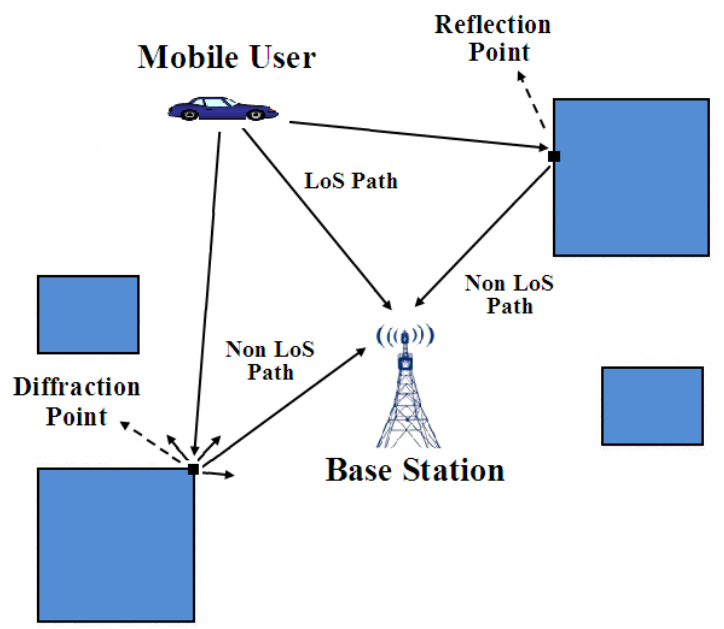

Figure 2. Diffraction phenomena and reflection phenomena (LoS and Non-LoS paths) for a 2D urban environment in reverse link

less than the number of sensors. Also, for simplicity we assume a synchronous DS-CDMA scheme and BPSK modulation in order to simplify the analysis of proposed technique. Additionally, in this paper we assume a slow fading channel (the channel random parameters do not change significantly during the bit interval). Hence, the received signal in the base station $q$ and sensor $s$ from all users can be written as [16], [20], [21]

$$
\begin{aligned}
r_{q, s}(t)=\sum_{k} & \sqrt{p_{k, m}^{\prime} \Gamma_{k}(x, y)} \sum_{l=1}^{L} \sqrt{\alpha_{k, m, l}} b_{k, m}\left(t-\tau_{k, m, l}\right) c_{k, m}\left(t-\tau_{k, m, l}\right) \\
& \times \exp \left(-j 2 \pi s d \sin \theta_{k, m, l} / \lambda\right)+n(t)
\end{aligned}
$$

where $c_{k, m}(t)$ is the pseudo noise (PN) chips of user $k$ in cell $m$ (user $k, m$ ) with a chip period of $T_{c} ; b_{k, m}(t)$ is the information bit sequence of user $k, m$ with a bit period of $T_{b}=G T_{c}$ where $G$ is processing gain; $\tau_{k, m, l}$ is the $l$ th path time delay for user $k, m ; \theta_{k, m, l}$ is the direction of arrival (DoA) in the $l$ th path for user $k, m ; \lambda$ is signal wavelength; $d$ is the distance between the antenna elements that for avoid the spatial aliasing should be defined as $d=0.5 \lambda ; n(t)$ is an additive white Gaussian noise (AWGN) process with a two-sided power spectral density (PSD) of $N_{0} / 2$. Also $\Gamma_{k}(x, y)$ is defined as

$$
\Gamma_{k}(x, y)=\left\{\begin{array}{c}
1 \quad ; k \in S_{\mathrm{BS} q} \\
\frac{\min _{m \in \Theta_{k}}\left\{1 / G_{k, m}\right\}}{1 / G_{k, q}} ; k \in S_{o}
\end{array}\right.
$$

where $G_{k, m}$ and $G_{k, q}$ are the best link gain between user $k$ and BS $m$ and BS $q$, respectively. It should be mentioned that in USPS, the channel is modeled as a lognormal distributed shadowing with mean 0 and variance $\sigma_{\xi}^{2}$, thus, the link gains are the function of $\sigma_{\xi}^{2}$. Also the variable $\Theta_{k}$ defined the set of the nearest BSs to user $k$ and $S_{\mathrm{BS} q}$ is the set of users that connected to $\operatorname{BS} q$ and $S_{o}$ is the set of users that not connected to $\operatorname{BS} q$ [2]. Also in Eq. (1), 
$\alpha_{k, m, l}$ is the normalized attenuation in USPS by the best link gain $\left(G_{k, m}\right)$ between user $k$ and BS $m$ in the $l$ th path, therefore $0<\alpha_{k, m, l} \leq 1$. Also in Eq. (1)

$$
p_{k, m}^{\prime}=G_{k, m} p_{k, m}
$$

is the received power in the $\mathrm{BS} m$ of user $k, m$ in the presence of closed-loop power control where $p_{k, m}$ is the transmitted power of user $k, m$ that in the case of the PPC, $p_{k, m}^{\prime}$ is fixed for all users within cell $m\left(p_{k, m}^{\prime}=P=E_{b} / T_{b}\right.$ where $E_{b}$ is the energy per bit for all users).

Accordingly, the received signal in the base station $q$ in sensor $s$ for user $i, q$ is given by [16]

$$
r_{i, q, s}^{\prime}(t)=\sum_{l=1}^{L} \sqrt{p_{i, q}^{\prime} \alpha_{i, q, l}} b_{i, q}\left(t-\tau_{i, q, l}\right) c_{i, q}\left(t-\tau_{i, q, l}\right) \exp \left(-j 2 \pi s d \sin \theta_{i, q, l} / \lambda\right)+I_{i, q, s}(t)+n(t)
$$

where $I_{i, q, s}(t)$ is the interference for user $i, q$ in sensor $s$ and can be shown to be

$$
\begin{aligned}
I_{i, q, s}(t)= & \sum_{m=1}^{M} \sum_{\substack{k=1 \\
k, m \neq i, q}}^{K_{m}} \sum_{l=1}^{L} \sqrt{p_{k, m}^{\prime} \alpha_{k, m, l} \Gamma_{k}(x, y)} b_{k, m}\left(t-\tau_{k, m, l}\right) c_{k, m}\left(t-\tau_{k, m, l}\right) \\
& \quad \times \exp \left(-j 2 \pi s d \sin \theta_{k, m, l} / \lambda\right)
\end{aligned}
$$

where $K_{m}$ is the number of users in cell $m$ and $M$ is the number of base stations/cells.

\section{Rake Receiver Performance Analysis}

The RAKE receiver structure in the DS-CDMA system is shown in Figure 1. The received signal is spatially processed by a beamforming circuit with the CG adaptive beamforming (CGBF) algorithm, one for each resolvable path ( $L$ beamformers). The resultant signal is then passed on to a set of parallel matched filters (MFs), on a finger-by-finger basis. Also, the output signals from the $L$ matched filters are combined and then are fed into the decision circuit for the desired user.

\subsection{Conjugate Gradient Adaptive Beamforming}

It is well known that an array of $N$ weights has $N-1$ degree of freedom for adaptive beamforming. This means that with an array of $N$ weights, one can generates $N-1$ pattern nulls and a beam maximum in desired directions. From Eq. (5), it is clear that the number of

users is $K_{u}=\sum_{m=1}^{M} K_{m}$ and the number of interference signals is $L K_{u}-1$. To null all of these interference signals; one would have to have $L K_{u}$ weights, which is not practical. So, we focus only on the $L$ paths of the desired user (inter-path interference). Thus, the minimum number of the antenna array weights is $L$ where, typically, $L$ varies from 2 to 6 [20], [21].

In this paper, we use the CGBF algorithm that is used of orthogonal principle [16], [17]. On this basis, a set of vectors $\mathbf{w}_{i}$ is to select such that they are $\mathbf{A}$-orthogonal, i.e., $\left\langle\mathbf{A} \mathbf{w}_{i}, \mathbf{A} \mathbf{w}_{j}\right\rangle=0$ for $i \neq j$. The optimum weights at time $n$ are obtained by minimizing [16]

$$
\left\|\mathbf{x}_{i, q}^{(j)}(n)\right\|^{2}=\mathbf{x}_{i, q}^{H(j)}(n) \mathbf{x}_{i, q}^{(j)}(n)
$$

where 
International Journal of Computer Networks \& Communications (IJCNC) Vol.2, No.6, November 2010

$$
\mathbf{x}_{i, q}^{(j)}(n)=\mathbf{A}_{q} \mathbf{w}_{i, q}^{(j)}(n)-\mathbf{y}_{i, q}^{(j)}
$$

and

$$
\mathbf{A}_{q}=\left[\begin{array}{ccc}
r_{q,-(N-1)} & \ldots & r_{q, 0} \\
\cdot & \cdot & \cdot \\
r_{q, 0} & \ldots & r_{q,+(N-1)}
\end{array}\right]
$$

is the $N \times N$ signal matrix in the base station $q$. Also,

$$
\mathbf{y}_{i, q}^{(j)}=\left[e^{-j(N-1) \theta_{i, q, j} / 2} \ldots 1 \ldots e^{+j(N-1) \theta_{i, q, j} / 2}\right]^{T}
$$

and

$$
\mathbf{w}_{i, q}^{(j)}(n)=\left[w_{i, q, 0}^{(j)}(n) w_{i, q, 1}^{(j)}(n) \quad \ldots \quad w_{i, q, N-1}^{(j)}(n)\right]^{T}
$$

are the excitation and weight vectors $(N \times 1)$ for user $i, q$ in the $j$ th path, respectively.

It should be mentioned that CG algorithm has two main characteristics [16]:

1- This algorithm can produce a solution of the matrix equation very efficiently and converge in a finite number of iterations (the number of beamformer weights).

2- In this algorithm, the convergence is guaranteed for any possible condition of the signal matrix, according to Eq. (8).

According to the algorithm of CG, the updated value of the weight vector for user $i, q$ in the $j$ th path at time $n+1$ is computed by using the simple recursive relation [16], [17]:

$$
\mathbf{w}_{i, q}^{(j)}(n+1)=\mathbf{w}_{i, q}^{(j)}(n)+\kappa_{i, q}^{(j)}(n) \boldsymbol{\beta}_{i, q}^{(j)}(n)
$$

where

$$
\begin{aligned}
& \kappa_{i, q}^{(j)}(n)=\left\|\mathbf{A}_{q}^{H} \mathbf{x}_{i, q}^{(j)}(n)\right\|^{2} /\left\|\mathbf{A}_{q} \boldsymbol{\beta}_{i, q}^{(j)}(n)\right\|^{2} \\
& \mathbf{x}_{i, q}^{(j)}(n+1)=\mathbf{x}_{i, q}^{(j)}(n)+\kappa_{i, q}^{(j)}(n) \boldsymbol{\beta}_{i, q}^{(j)}(n) \\
& \boldsymbol{\beta}_{i, q}^{(j)}(0)=-\mathbf{A}_{q}^{H} \mathbf{x}_{i, q}^{(j)}(0) \\
& \boldsymbol{\beta}_{i, q}^{(j)}(n+1)=\mathbf{A}_{q}^{H} \mathbf{x}_{i, q}^{(j)}(n+1)+\eta_{i, q}^{(j)}(n) \boldsymbol{\beta}_{i, q}^{(j)}(n) \\
& \eta_{i, q}^{(j)}(n)=\left\|\mathbf{A}_{q}^{H} \mathbf{x}_{i, q}^{(j)}(n+1)\right\|^{2} /\left\|\mathbf{A}_{q}^{H} \mathbf{x}_{i, q}^{(j)}(n)\right\|^{2}
\end{aligned}
$$

The output signal from the $j$ th CG beamformer $(j=1, \ldots, L)$ can be written as

$$
y_{i, q}^{(j)}(t)=\sqrt{p_{i, q}^{\prime} \alpha_{i, q, j}} b_{i, q}\left(t-\tau_{i, q, j}\right) c_{i, q}\left(t-\tau_{i, q, j}\right)+I_{i, q}^{(j)}(t)+n^{(j)}(t)
$$

where $n^{(j)}(t)$ is a zero mean Gaussian noise of variance $\sigma_{n}^{2}$ and $I_{i, q}^{(j)}(t)$, the MAI, is defined as

$$
I_{i, q}^{(j)}(t)=\sum_{m=1}^{M} \sum_{\substack{k=1 \\ k, m \neq i, q}}^{K_{m}} \sum_{l=1}^{L} \sqrt{p_{k, m}^{\prime} \alpha_{k, m, l} \Gamma_{k}(x, y)} g_{i, q}^{(j)}\left(\theta_{k, m, l}\right) b_{k, m}\left(t-\tau_{k, m, l}\right) c_{k, m}\left(t-\tau_{k, m, l}\right)
$$

where 
International Journal of Computer Networks \& Communications (IJCNC) Vol.2, No.6, November 2010

$$
g_{i, q}^{(j)}(\theta)=\left[e^{-j(N-1) \theta / 2} \ldots 1 \ldots e^{+j(N-1) \theta / 2}\right] \times \mathbf{w}_{i, q}^{(j)}
$$

is the magnitude response of the $j$ th beamformer for user $i, q$ toward the direction of arrival $\theta$ and $\mathbf{w}_{i, q}^{(j)}$ is the $j$ th beamformer's weight vector for user $i, q$.

\subsection{Matched Filter Stage}

Using beamforming will only cancel out the IPI for the desired user and will reduce the MAI from the users whose signals arrive at different angles from the desired user signal (out-beam interference). Now, in the second stage of the RAKE receiver, the output signal from the $j$ th beamformer is directly passes on to a filter matched to the desired user's signature sequence. The $j$ th matched filter output corresponding to the $n$th bit is [16]:

$$
z_{i, q}^{(j)}(n)=\sqrt{p_{i, q}^{\prime} \alpha_{i, q, j}} b_{i, q}(n)+I_{i, q}^{\prime(j)}(n)+n^{(j)}(n)
$$

where

$$
I_{i, q}^{\prime(j)}(n)=\frac{1}{T_{b}} \int_{(n-1) T_{b}+\tau_{i, q, j}}^{n T_{b}+\tau_{i, q, j}} I_{i, q}^{(j)}(t) c_{i, q}\left(t-\tau_{i, q, j}\right) d t
$$

and

$$
n^{\prime(j)}(n)=\frac{1}{T_{b}} \int_{(n-1) T_{b}+\tau_{i, q, j}}^{n T_{b}+\tau_{i, q, j}} n^{(j)}(t) c_{i, q}\left(t-\tau_{i, q, j}\right) d t
$$

If we assume that the paths' delays from all users are less than the symbol duration $\left(\tau_{k, m, l}<T_{b}\right)$ for all users' signals on all paths, the $n$th bit MAI at the output of the $j$ th matched filter are expressed as

$$
I_{i, q}^{(j)}(n)=\sum_{m=1}^{M} \sum_{\substack{k=1 \\ k, m \neq i, q}}^{K_{m}} \sum_{l=1}^{L} \sqrt{p_{k, m}^{\prime} \alpha_{k, m, l} \Gamma_{k}(x, y)} g_{i, q}^{(j)}\left(\theta_{k, m, l}\right) b_{k, m}(n) R_{i, k}\left(\tau_{i, q, j}-\tau_{k, m, l}\right)
$$

where the autocorrelation function $R_{i, k}(\tau)$ is [16], [22]:

$$
R_{i, k}(\tau)=\frac{1}{T_{b}} \int_{T_{b}} c_{i, q}(t) c_{k, m}(t+\tau) d t
$$

If all users' delays are multiples of the chip period $\left(T_{c}\right)$, then

$$
R_{i, k}(\tau)=\frac{1}{G} \sum_{l_{1}=0}^{G-1} \sum_{l_{2}=0}^{G-1} c_{i, q}\left(l_{1}\right) c_{k, m}\left(l_{2}\right) R_{c}\left(\tau-\left(l_{1}-l_{2}\right) T_{c}\right)
$$

where the autocorrelation function $R_{c}(\tau)$ is:

$$
R_{c}(\tau)=\frac{1}{T_{b}} \int_{T_{b}} c(t) c(t+\tau) d t
$$

In the case of a maximal-length sequence (m-sequence) and for $0 \leq \tau \leq T_{b}$, we have [22]: 


$$
R_{c}(\tau)= \begin{cases}1-\frac{|\tau|}{T_{c}}(1+1 / G) & ;|\tau| \leq T_{c} \\ -1 / G & ;|\tau| \geq T_{c}\end{cases}
$$

Now, the SINR in output of the RAKE receiver for user $i, q$ is given as [16], [23]

$$
\operatorname{SINR}_{i, q}(\alpha)=\sum_{j=1}^{L} \operatorname{SINR}_{i, q}^{(j)}(\alpha)
$$

where

$$
\operatorname{SINR}_{i, q}^{(j)}(\alpha)=\frac{p_{i, q}^{\prime} \alpha_{i, q, j}}{\mathrm{E}\left(I_{i, q}^{\prime(j)}\right)^{2}+\mathrm{E}\left(n^{\prime(j)}\right)^{2}}
$$

is the SINR in output of the RAKE receiver in path $j$ for user $i, q$.

Now, we can be rewritten the SINR in Eq. (25) as follows.

$$
\operatorname{SINR}_{i, q}^{(j)}(\alpha)=\frac{p_{i, q}^{\prime} \alpha_{i, q, j}}{\sum_{m=1}^{M} \sum_{\substack{k=1 \\ k, m \neq i, q}}^{K_{m}} p_{k, m}^{\prime} \Gamma_{k}(x, y) \sum_{l=1}^{L} \alpha_{k, m, l}\left|g_{i, q}^{(j)}\left(\theta_{k, m, l}\right)\right|^{2} R_{i, k}^{2}\left(\tau_{i, q, j}-\tau_{k, m, l}\right)+\frac{N_{0}}{2 T_{b}}}
$$

In order to perform the BER, we assume Gaussian approximation for the probability density function of interference plus noise. The conditional BER for a BPSK modulation is [16], [22]:

$$
\operatorname{BER}_{i, q}(\alpha)=Q\left(\sqrt{2 \times \operatorname{SINR}_{i, q}(\alpha)}\right)
$$

where

$$
Q(x)=\frac{1}{\sqrt{2 \pi}} \int_{x}^{\infty} \exp \left(-u^{2} / 2\right) d u
$$

\section{Smart Step Closed-Loop Power Control Algorithm}

A major limiting factor for the satisfactory performance of CDMA systems is the near-far effect. Power control is an intelligent way of adjusting the transmitted powers in cellular systems so that the TTP is minimized, but at the same time, the user SINRs satisfies the system quality of service (QoS) requirements [24], [25].

Depending on the location where the decision on how to adjust the transmitted powers is made, the power control algorithm can be divided into two groups: centralized and distributed techniques [1]-[6], [20]. In centralized power control, a network center can simultaneously compute the optimal power levels for all users. However, it requires measurement of all the link gains and the communication overhead between a network center and base stations. Thus, it is difficult to realize in a large system [26]. Distributed power control, on the other hand, uses only local information to determine transmitter power levels. It is much more scalable than centralized power control. However, transmitter power levels may not be optimal, resulting in performance degradation [27], [28].

The distributed closed-loop power control problem has been investigated by many researchers from many perspectives during recent years [4], [24], [27]-[29]. For instance, the conventional fast closed-loop power control strategy used in practice in CDMA systems is a fixed step 
controller based on SINR measurements. The fixed step closed-loop power control (FSPC) algorithm is defined by [4]

$$
p_{i, q}^{n^{\prime}+1}=p_{i, q}^{n^{\prime}}+\delta \operatorname{sign}\left(\gamma_{i, q}^{*}-\gamma_{i, q}^{n^{\prime}}\right)
$$

where $p_{i, q}^{n^{\prime}}, \gamma_{i, q}^{*}$, and $\gamma_{i, q}^{n^{\prime}}$ are the transmitter power, SINR target, and measured SINR of user $i, q$ at time $n^{\prime}$, respectively, and $\delta$ is the fixed step size. Also $p_{i, q}^{n^{\prime}+1}$ is transmitter power control (TPC) command in the feedback link of the base station to user $i, q$ at time $n^{\prime}+1$ (all signals are in decibels).

Also, the distributed traditional closed-loop power control (DTPC) is defined by [24]

$$
p_{i, q}^{n^{\prime}+1}=\frac{\gamma_{i, q}^{*}}{\gamma_{i, q}^{n^{\prime}}} p_{i, q}^{n^{\prime}}
$$

In both algorithms, the simple intuition behind this iteration is that if the current SINR $\gamma_{i, q}^{n^{\prime}}$ of user $i, q$ is less than the target $\operatorname{SINR} \gamma_{i, q}^{*}$, then the power of that user is increased; otherwise, it is decreased.

It should be mentioned that convergence speed of DTPC algorithm is higher than FSPC algorithm. Also, the variance of the SINR mis-adjustment in FSPC algorithm is higher than DTPC algorithm. But, it has been shown that the FSPC algorithm converges to $\left|\gamma_{i, q}^{*}-\gamma_{i, q}^{n^{\prime}}\right| \leq 2 \delta k_{d}$, where $k_{d}$ is the loop delay [3].

Also in [29], variable step closed-loop power control (VSPC) algorithm has been proposed. In this algorithm, variable step size is discrete with mode $q_{v}$. It is shown that the performance of VSPC algorithm with mode $q_{v}=4$ is found to be worse than that of a fixed step algorithm ( $\left.q_{v}=1\right)$ under practical situations with loop delay of two power control intervals, but the convergence speed of VSPC algorithm is higher than FSPC algorithm. Also in this algorithm, the variance of the SINR mis-adjustment is reduced in compared to FSPC algorithm.

Practical implementations of power control in CDMA systems utilize closed-loop control, where the transmitter adjusts its power based on commands received from the receiver in a feedback channel. To minimize signaling overhead, typically one bit is used for the power control command. In practice, the command must be derived based on measurements made at the receiver, transmitted over the feedback channel to the transmitter, and finally processed and applied at the transmitter. All these operations constitute a loop delay, which can cause problems if it is not properly taken care of in the design of the power control algorithm. In many cases the loop delay is known due to a specific frame structure inherent in the system. A typical loop delay situation encountered in wideband CDMA (WCDMA) systems is shown in Figure 3. The slot at time $n^{\prime} t$ is transmitted using power $p^{n^{\prime}}$. The receiver measures the SINR $\gamma^{n^{\prime}}$ over a number of pilot and/or data symbols and derives a TPC command. The command is transmitted to the transmitter in the feedback link and the transmitter adjusts its power at time $\left(n^{\prime}+1\right) t$ according to the command. It should be mentioned that since the power control signaling is standardized, the loop delays are known exactly [4].

In this paper, we propose the smart step closed-loop power control algorithm. We express the SSPC algorithm as follows [7]-[10], [13], [14]. 
International Journal of Computer Networks \& Communications (IJCNC) Vol.2. No.6. November 2010

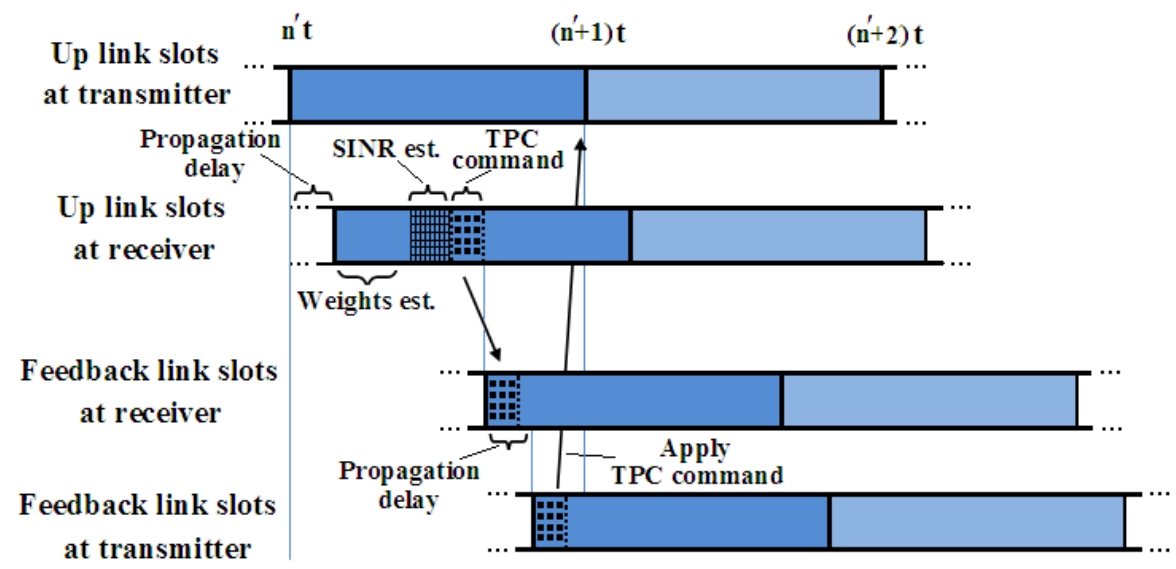

Figure 3. Example of power control timing in WCDMA systems [4]

$$
p_{i, q}^{n^{\prime}+1}=p_{i, q}^{n^{\prime}}+\delta\left|\gamma_{i, q}^{*}-\gamma_{i, q}^{n^{\prime}}\right| \operatorname{sign}\left(\gamma_{i, q}^{*}-\gamma_{i, q}^{n^{\prime}}\right)
$$

The SSPC algorithm is implemented as follows.

1) Select the initial transmitted power vector $\left(n^{\prime}=0\right)$ for all users within cell $m$ as

$$
\mathbf{p}_{m}^{0}=\left[\begin{array}{lll}
p_{1, m}^{0} & p_{2, m}^{0} \ldots & p_{K_{m}, m}^{0}
\end{array}\right], m=1,2, \ldots, M .
$$

2) Estimate the weight vector for all users with the CG algorithm using Eq. (11).

3) Calculate the SINR for all users using Eq. (24).

4) If $\left|\gamma_{k, m}^{*}-\gamma_{k, m}^{n^{\prime}}\right|>\varepsilon_{0}$ for each user then set $n^{\prime}=n^{\prime}+1$ and calculate the TPC for all users at time $n^{\prime}+1$ using Eq. (31) and go back to 2), where $\varepsilon_{0}$ is threshold value.

5) Finally, if $\left|\gamma_{k, m}^{*}-\gamma_{k, m}^{n^{\prime}}\right|<\varepsilon_{0}$ for all users then algorithm ends.

As will be seen from simulation results, because of variable coefficient in the sign function, the convergence speed of our algorithm is higher than FSPC and VSPC algorithms.

\section{Power Control Error}

When imperfections in power control are considered, multipath fading is not perfectly compensated. As a result, the power received from a mobile will not be constant at the base station to which the mobile is connected. Accordingly, we can be rewritten Eq. (1) as follows.

$$
r_{q, s}(t)=\sum_{k} \sqrt{P \lambda_{k, m} \Gamma_{k}(x, y)} \sum_{l=1}^{L} \sqrt{\alpha_{k, m, l}} b_{k, m}\left(t-\tau_{k, m, l}\right) c_{k, m}\left(t-\tau_{k, m, l}\right) \exp \left(-j s k_{d} \sin \theta_{k, m, l}\right)+n(t)
$$

where $P=E_{b} / T_{b}$ represents the received signal power of all users within cell $q$ in the presence of PCE. The variable $\lambda_{k, m}$ is PCE for user $k, m$, which is assumed to follow a log-normal distribution and thus it can be written as $\lambda_{k, m}=10^{v_{k, m} / 10}$, where $v_{k, m}$ is a Gaussian random variable with mean 0 and variance $\sigma_{v}^{2}$ for all users [2]. On the other hand, $\mathrm{E}\left[\lambda_{k, m}\right]$ for all users can be written as follows [30]. 


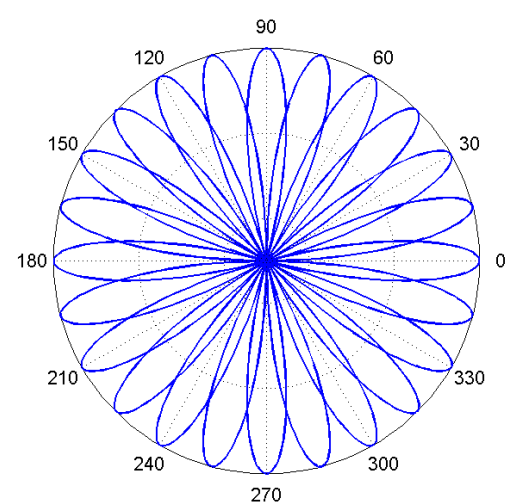

Figure 4. 36 beams in each base station with the SB technique [15]

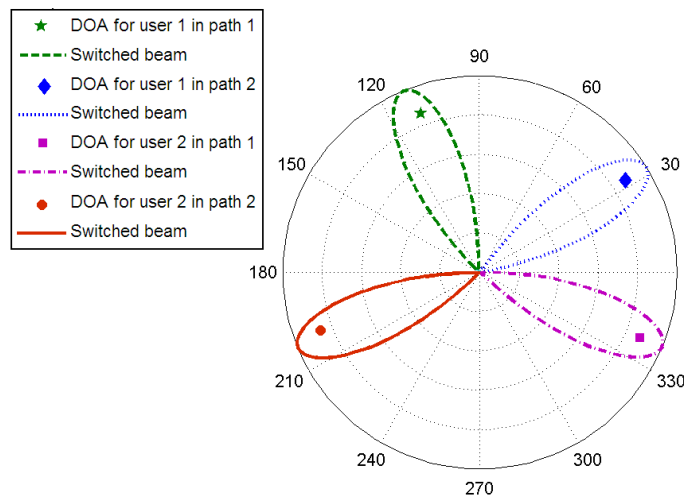

Figure 5. Select of beam for two users in two paths with the SB technique [15]

$$
\mathrm{E}\left[\lambda_{k, m}\right]=e^{\beta^{2} \sigma_{v}^{2} / 2}
$$

where $\beta=\ln (10) / 10$. Accordingly, we can be rewritten the SINR in Eq. (25) as follows.

$$
\operatorname{SINR}_{i, q}^{(j)}(\alpha)=\frac{P e^{\beta v_{i, q}} \alpha_{i, q, j}}{\mathrm{E}\left(I_{i, q}^{\prime(j)}\right)^{2}+\mathrm{E}\left(n^{\prime(j)}\right)^{2}}
$$

Also using Eq. (26) and Eq. (33), we can be rewritten the SINR in Eq. (34) as follows [11], [31].

$$
\operatorname{SINR}_{i, q}^{(j)}(\alpha)=\frac{e^{\beta v_{i, q}} \alpha_{i, q, j}}{e^{\beta^{2} \sigma_{v}^{2} / 2} \sum_{\substack{m=1 \\ k, m \neq i, q}}^{M} \sum_{\substack{k=1 \\ k}}^{K_{m}} \Gamma_{k}(x, y) \sum_{l=1}^{L} \alpha_{k, m, l}\left|g_{i, q}^{(j)}\left(\theta_{k, m, l}\right)\right|^{2} R_{i, k}^{2}\left(\tau_{i, q, j}-\tau_{k, m, l}\right)+\frac{0.5}{E_{b} / N_{0}}}
$$

\section{THE SWITCHED-BEAM TECHNIQUE}

One simple alternative to the fully adaptive antenna is the switched-beam architecture in which the best beam is chosen from a number of fixed steered beams. Switched-beam systems are technologically the simplest and can be implemented by using a number of fixed, independent, or directional antennas [32].

We list the conditions of the SB technique for this paper as follows [13]-[15].

1) According to Figure 4, beams coverage angle is $30^{\circ}$ and overlap between consecutive beams is $20^{\circ}$. Thus each base station has 36 beams.

2) According to Figure 5, each user can be use one beam for its each path to communicate with a base station at any time.

One simple method to sectorize a cell is equal sectoring; in which all sectors have the same coverage angle. In this paper, we assume three sectors for each base station with sector angle $120^{\circ}$ for the ES method. 


\section{Simulation Results}

We consider $M=9$ base stations for a nine-cell DS-CDMA system as Figure 6 [33]. We assume a uniform linear array of $S$ omni-directional antennas in each base station with antenna spacing $d=\lambda / 2$. Also, we assume the input data rate $T_{b}=9.6 \mathrm{Kbps}$; the number of antenna weights $N=3$; the number of antenna sensors $S=5 ; L=4$ resolvable propagation paths for all users; resolution, path loss parameter, and variance of the log-normal shadow fading in

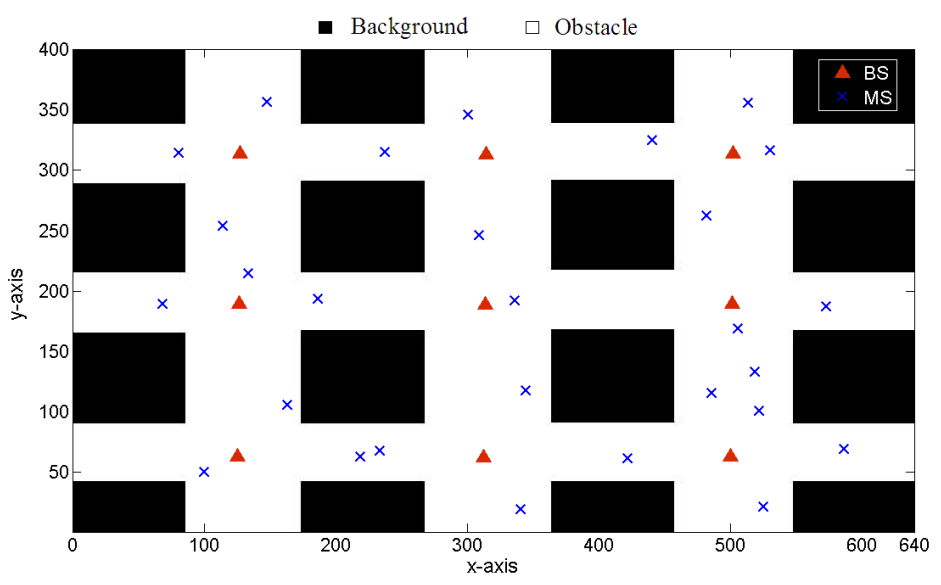

Figure 6. Placing users and $M=9$ base stations in a two-dimensional map for USPS [33]

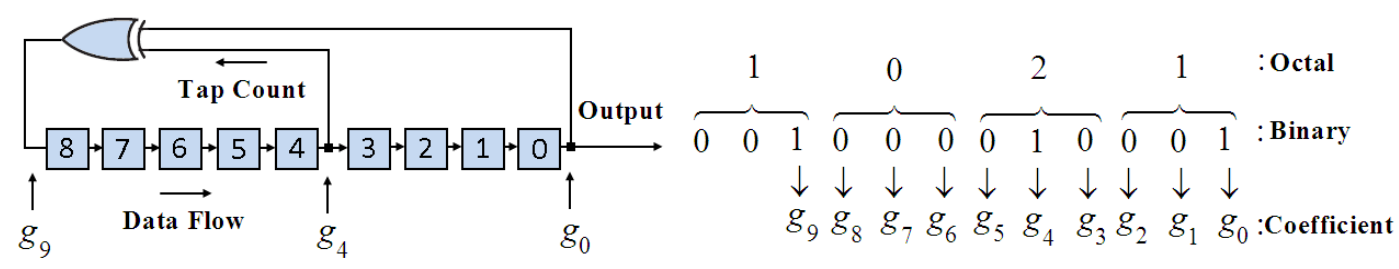

(a)

(b)

Figure 7. (a) Fibonacci feedback generator for LFSR polynomial $g(D)=1+D^{4}+D^{9}$ for ninestage shift register (b) Expanding the octal entry 1021 into binary form [22]

USPS $R=1, L_{p}=0.05 \mathrm{~dB} / \mathrm{m}$, and $\sigma_{\xi}^{2}=4 \mathrm{~dB}$ respectively; initial value for weight vectors in the CGBF algorithm $\mathbf{w}(0)=\mathbf{0}$. The SINR target value is the same for all users and is set to $\gamma^{*}=10(10 \mathrm{~dB})$. It also is assumed that the distribution of users in all cells is uniform.

In this paper, we use m-sequence generator with processing gain $G=512$ based on linear feedback shift register (LFSR) circuit using the Fibonacci feedback approach [22]. This structure is shown in Figure 7 (a). Also, according to [22], we use the sequence generated by the polynomial corresponding to the entry the octal representation of generator polynomial, ORGP= [1021]* for a nine-stage shift register. Figure 7 (b) shows expanding the octal entry 1021 into binary form. Then, the LFSR polynomial is $g(D)=1+D^{4}+D^{9}$.

Figure 8 shows the comparison of the average SINR achieved over $K_{u}=120$ users and signal to noise ratio, $\mathrm{SNR}=10 \mathrm{~dB}$, versus the power control iteration index $\left(n^{\prime}\right)$ for SSPC, VSPC $\left(q_{v}=4\right.$ ), and FSPC algorithms. In this simulation, the two-stage RAKE receiver uses CGBF, SB, or ES methods in the first stage. Also, we assume that each user to have a maximum power constraint of 1watt. Accordingly, we observe that the convergence speed of the SSPC algorithm is faster 
than the VSPC and FSPC algorithms. For example, the SSPC algorithm with CGBF algorithm converges in about 11 iterations, while VSPC and FSPC algorithms converge in about 14 and 19 iterations, respectively. In addition, we see that the convergence speed of the SSPC algorithm for the SB technique is faster than the CGBF algorithm and ES method. Also observe that the average SINR level achieved is below the target SINR value for the ES method, because in this method the MAI is higher than CGBF algorithm and SB technique.

Figure 9 shows the comparison of TTP usage versus the power control iteration index $\left(n^{\prime}\right)$, as Figure 8 . But in this simulation, we assume that users no have maximum power constraints.

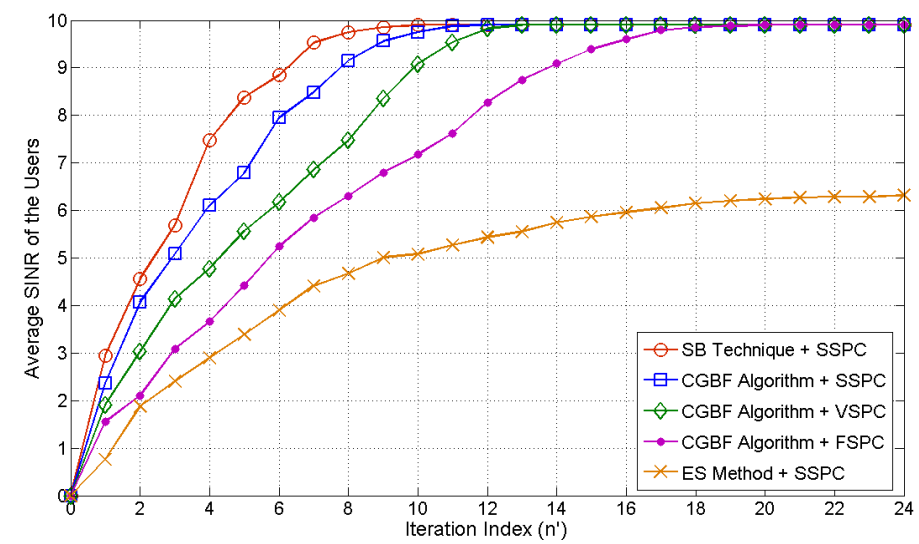

Figure 8. Average SINR of all users versus power control iteration index $\left(n^{\prime}\right)$, with maximum power constraint of $1 \mathrm{watt}, K_{u}=120$, and $\mathrm{SNR}=10 \mathrm{~dB}$

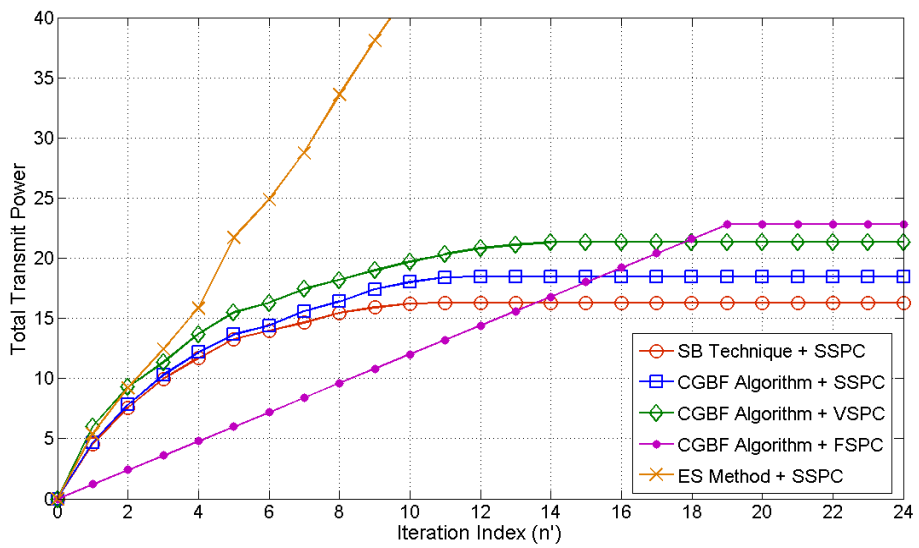

Figure 9. Total transmit power of all users versus power control iteration index $\left(n^{\prime}\right), K_{u}=120$, and $\mathrm{SNR}=10 \mathrm{~dB}$. No power constraints

Similar to Figure 8, we observe that the ES method never can achieve the target SINR value for all users. Also this figure shows that the SSPC algorithm offers more savings in the TTP as compared the FSPC and VSPC algorithms. In addition, we see that the TTP for the joint SSPC algorithm and SB technique is lower than other cases. For example with the SSPC algorithm, TTP for the SB technique is 16.3 watt, while for the CGBF algorithm is 18.45 watt.

Figure 10 shows the average BER versus SNR for different receivers (one and two-stage receivers), $K_{u}=120$ active users, and different values of $\sigma_{v}^{2}$. It is clear that, in MF only 
International Journal of Computer Networks \& Communications (IJCNC) Vol.2, No.6, November 2010

receiver (one-stage receiver), we still have the error floor at high SNR. Using CGBF and MF receiver (two-stage RAKE receiver as Figure 1) or SB and MF receiver, has a better performance than using MF only. In addition, the figure shows that the average BER in the CGBF algorithm is higher than the SB technique. For example, at a SNR of $8 \mathrm{~dB}$ and $\sigma_{v}^{2}=0 \mathrm{~dB}$ (perfect power control), the average BER is 0.0059 for the CGBF technique, while for SB technique the average BER is 0.00075 . Also it can be seen that the average BER for case of PPC is lower than $\sigma_{v}^{2}=4 \mathrm{~dB}$ [7]-[10]. For example, at a SNR of $10 \mathrm{~dB}$ and for CGBF and MF receiver, the average BER is 0.0042 for $\sigma_{v}^{2}=0 \mathrm{~dB}$, while for $\sigma_{v}^{2}=4 \mathrm{~dB}$ average BER is 0.0062 .

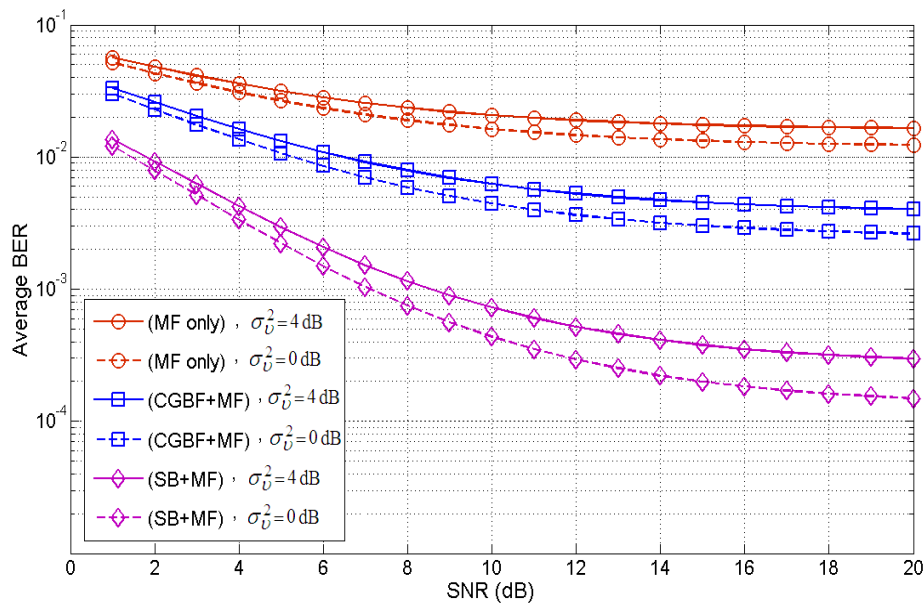

Figure 10. Average BER of all users versus the SNR for $K_{u}=120$ and different values of $\sigma_{v}^{2}$

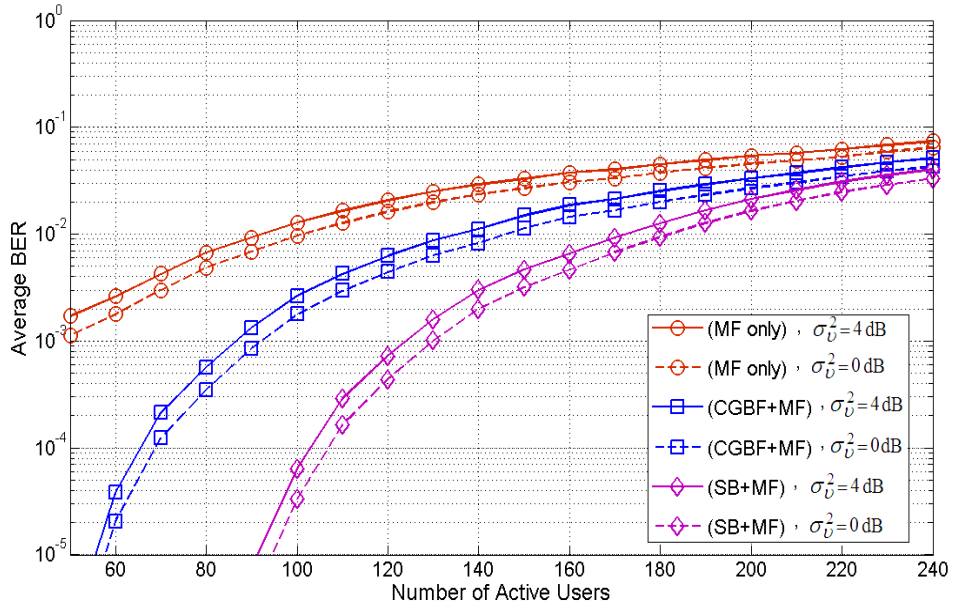

Figure 11. Average BER versus $K_{u}$ for $\mathrm{SNR}=10 \mathrm{~dB}$ and for different values of $\sigma_{v}^{2}$ 
Finally, Figure 12 shows the influence of path-loss parameter in USPS $\left(L_{p}\right)$ on the average BER for $\sigma_{v}^{2}=4 \mathrm{~dB}$ and $\mathrm{SNR}=10 \mathrm{~dB}$. We can observe that, as expected, a decrease in the pathloss parameter entails an increase in the interference and desired signal levels and, therefore using antenna arrays in BSs, an improvement in system performance. For example, at a BER of 0.01 , capacity is, respectively, 105,136 , and 201 users for $L_{p}=0.5,0.05$, and $0.01 \mathrm{~dB} / \mathrm{m}$. Thus it is seen that if $L_{p}$ decreases from 0.5 to $0.01 \mathrm{~dB} / \mathrm{m}$, the number of active users increases by approximately $48 \%$.

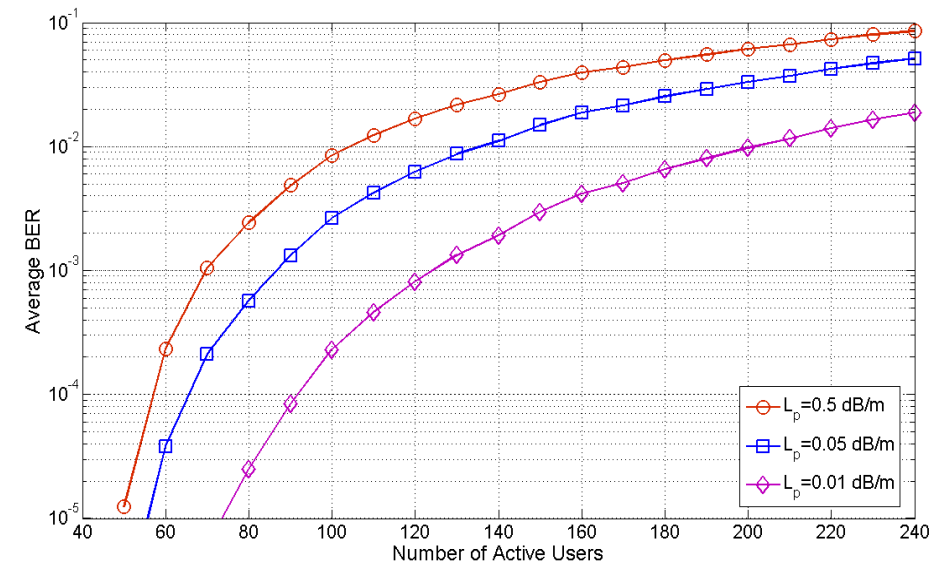

Figure 12. Influence of path-loss parameter on average BER $\left(\sigma_{v}^{2}=4 \mathrm{~dB}, \mathrm{SNR}=10 \mathrm{~dB}\right)$

\section{Conclusions}

In this paper, we studied performance of DS-CDMA cellular systems in a 2D urban environment with closed-loop power control and adaptive beamforming. In this paper, we determined the optimum weights of the elements of array antenna with the CGBF algorithm. Accordingly, we proposed the SSPC algorithm in order to compensate the effects of the near-far problem. It has been shown that, by using antenna arrays at BSs, this algorithm will decrease the interference in all cells. In addition, the TTP expected by all users is less as compared to the VSPC and FSPC algorithms. Thus, it decreases the BER by allowing the SINR targets for the users to be higher, or by increasing the number of users supportable at a fixed SINR target level. It has also been observed that the TTP in SB technique is less than CGBF algorithm. Also, it has been shown that the convergence speed of the proposed algorithm is increased in comparison with the VSPC and FSPC algorithms. Also, we see that the convergence speed of the SSPC algorithm with the SB technique is faster than the CGBF algorithm and ES method. In addition, our simulations show that the variations in power level due to PCE have a detrimental effect on system performance.

\section{ACKNOWLEDGEMENT}

This research is supported under research project by the Islamic Azad University, Qazvin Branch, Qazvin, Iran. 
International Journal of Computer Networks \& Communications (IJCNC) Vol.2, No.6, November 2010

\section{REFERENCES}

[1] A. Abrardo and D. Sennati, "On the analytical evaluation of closed-loop power-control error statistics in DS-CDMA cellular systems," IEEE Transactions on Vehicular Technology, vol. 49, no. 6, pp. 2071-2080, Nov. 2000.

[2] L. Carrasco and G. Femenias, "Reverse link performance of a DS-CDMA system with both fast and slow power controlled users," IEEE Transactions on Wireless Communications, vol. 7, no. 4, pp. 1255-1263, Apr. 2008.

[3] L. Qian and Z. Gajic, "Variance minimization stochastic power control in CDMA system," IEEE Transactions on Wireless Communications, vol. 5, no. 1, pp. 193-202, Jan. 2006.

[4] M. Rintamaki, H. Koivo, and I. Hartimo, "Adaptive closed-loop power control algorithms for CDMA cellular communication systems," IEEE Transactions on Vehicular Technology, vol. 53, no. 6, pp. 1756-1768, Nov. 2004.

[5] J. Wang and A. Yu, "Open-loop power control error in cellular CDMA overlay systems," IEEE Journal on Selected Areas in Communications, vol. 19, no. 7, pp. 1246-1254, July 2001.

[6] J. T. Wang, "Admission control with distributed joint diversity and power control for wireless networks," IEEE Transactions on Vehicular Technology, vol. 58, no. 1, pp. 409-419, Jan. 2009.

[7] M. Dosaranian-Moghadam, H. Bakhshi, and G. Dadashzadeh, "Interference management for DS-CDMA systems through closed-loop power control, base station assignment, and beamforming," Journal of Wireless Sensor Network, vol. 2, no. 6, pp. 472-482, June 2010.

[8] M. Dosaranian-Moghadam, H. Bakhshi, and G. Dadashzadeh, "Adaptive beamforming method based on closed-loop power control for DS-CDMA receiver in multipath fading channel," Accepted for publication in the $21^{\text {th }}$ IEEE Symposium on Personal, Indoor, and Mobile Radio Communications, Istanbul, Turkey, Sep. 26-30, 2010.

[9] M. Dosaranian-Moghadam, H. Bakhshi, G. Dadashzadeh, and M. Godarzvand-Chegini, "Joint closed-loop power control and constrained LMS algorithm for DS-CDMA receiver in multipath fading channels," Accepted for publication in IEEE Global Mobile Congress, Shanghai, China, Oct. 18-19, 2010.

[10] M. Dosaranian-Moghadam, H. Bakhshi and G. Dadashzadeh, Joint closed-loop power control and base station assignment for DS-CDMA receiver in multipath fading channel with Adaptive Beamforming Method, Iranian Journal of Electrical and Electronic Engineering, vol. 6, no.3, pp. 156-167, Sep. 2010.

[11] M. Dosaranian-Moghadam, H. Bakhshi, G. Dadashzadeh, and M. Godarzvand-Chegini, "Joint base station assignment, power control error, and adaptive beamforming for DS-CDMA cellular systems in multipath fading channels," Accepted for publication in IEEE Global Mobile Congress, Shanghai, China, Oct. 18-19, 2010.

[12] M. Dosaranian-Moghadam, H. Bakhshi, G. Dadashzadeh, "DS-CDMA cellular systems performance with base station assignment, power control error, and beamforming over multipath fading," Accepted for publication in International Journal of Computer Networks \& Communications.

[13] M. Dosaranian-Moghadam, H. Bakhshi, G. Dadashzadeh, "Joint closed-loop power control and adaptive beamforming for wireless networks with antenna arrays in a 2D Urban Environment," Accepted for publication in Journal of Wireless Sensor Network.

[14] M. Dosaranian-Moghadam, H. Bakhshi, G. Dadashzadeh, "Closed-loop power control in wireless networks using constrained LMS algorithm in a 2-D urban environment," Accepted for publication in the IEEE International Conference on Communication Systems, Singapore, Nov. 17-20, 2010.

[15] M. Dosaranian-Moghadam, H. Bakhshi, and G. Dadashzadeh, "Joint centralized power control and cell sectoring for interference management in CDMA cellular systems in a 2D urban environment," Journal of Wireless Sensor Network, vol. 2, no. 8, pp. 599-605, Aug. 2010. 
International Journal of Computer Networks \& Communications (IJCNC) Vol.2, No.6, November 2010

[16] N. A. Mohamed and J. G. Dunham, "A low-complexity combined antenna array and interference cancellation DS-CDMA receiver in multipath fading channels," IEEE Journal on Selected Areas in Communications, vol. 20, no. 2, pp. 248-256, Feb. 2002.

[17] N. A. Mohamed and J. G. Dunham, "Adaptive beamforming for DS-CDMA using conjugate gradient algorithm in a multipath fading channel," in Proc. 1999 IEEE Emerging Technologies Symp., Dallas, TX, pp. 859-863, Apr. 12-13, 1999.

[18] W. Ye and A. M. Haimovich, "Performance of cellular CDMA with cell site antenna array, Rayleigh fading, and power control error," IEEE Transactions on Communications, vol. 48, no. 7, pp. 1151-1159, July 2000.

[19] M. Dosaranian-Moghadam, H. Bakhshi, G. Dadashzadeh, and P. Rahmati, "Adaptive beamforming method based on constrained LMS algorithm for tracking mobile user," IEEE Global Mobile Congress, Shanghai, China, pp. 1-6, Oct. 2009.

[20] F. Rashid-Farrokhi, L. Tassiulas, and K. J. Ray-Liu, "Joint optimal power control and beamforming in wireless networks using antenna arrays," IEEE Transactions on Communications, vol. 46, no. 10, pp. 1313-1324, Oct. 1998.

[21] J. Litva and T. Kwok-Yeung, Digital Beamforming in Wireless Communications. Artech-House, 1996.

[22] R. L. Peterson, R. E. Ziemer, and D. E. Borth, Spread-Spectrum Communications. Prentice-Hall, 1995.

[23] N. Kong and L. B. Milstein, "Average SNR of a generalized diversity selection combining scheme,” IEEE Communications Letters, vol. 3, no. 3, pp. 57-59, Mar. 1999.

[24] A. Yener, R. D. Yates, and S. Ulukus, "Interference management for CDMA systems through power control, multiuser detection, and beamforming," IEEE Transactions on Communications, vol. 49, no. 9, pp. 1227-1239, July 2001.

[25] S. Kandukuri and S. Boyd, "Optimal power control in interference-limited fading wireless channels with outage probability specifications," IEEE Transactions on Wireless Communications, vol. 1, no. 1, pp. 46-55, Jan. 2002.

[26] S. Grandhi, R. Vijayan, and D. Goodman, "Centralized power control in cellular radio systems," IEEE Transactions on Vehicular Technology, vol. 42, no. 4, pp. 466-468, Nov. 1993.

[27] J. Zander, "Distributed cochannel interference control in cellular radio systems," IEEE Transactions on Vehicular Technology, vol. 44, pp. 305-311, Aug. 1992.

[28] S. Grandhi, R. Vijayan, and D.Goodman, "Distributed power control in cellular radio systems," IEEE Transactions on Communications, vol. 42, no. 2/3/4, pp. 226-228, Feb/Mar/Apr. 1994.

[29] A. Kurniawan, "Effect of feedback delay on fixed step and variable step power control algorithm in CDMA systems," IEEE International Conference on Communication Systems, Singapore, vol. 2, pp. 1096-1100, Nov. 2002.

[30] J. M. Romero-Jerez, C. Tellez-Labao, and A. Diaz-Estrella, "Effect of power control imperfections on the reverse link of cellular CDMA networks under multipath fading," IEEE Transactions on Vehicular Technology, vol. 53, no.1, pp. 61-71, Jan. 2004.

[31] J. C. Liberti and T. S. Rappaport, Smart Antennas for Wireless Communications IS -95 and Third Generation CDMA Applications. Prentice-Hall, 1999.

[32] B. Allen and M. Beach, "On the analysis of switched-beam antennas for the W-CDMA downlink”, IEEE Transactions on Vehicular Technology, vol. 53, no. 3, pp. 569-578, May 2004.

[33] M. Dosaranian-Moghadam, H. Bakhshi, G. Dadashzadeh, "Performance of DS-CDMA Systems with Conjugate Gradient Adaptive Beamforming and Base Station Assignment in a 2D Urban Environment," Accepted for publication in the IEEE International Symposium on Telecommunication, Iran, Tehran, Dec. 4-6, 2010. 


\section{Authors}

Mohamad Dosaranian-Moghadam was born in Tehran, Iran on May 26, 1979. $\mathrm{He}$ received the B.Sc. degree in electrical engineering from Islamic Azad University, Qazvin Branch, Qazvin, Iran and the M.Sc. degree in communication engineering from Ferdowsi University, Mashad, Iran, in 2002 and 2005, respectively. He is currently working toward the Ph.D degree in the Department of Electrical Engineering, Islamic Azad University, Science and Research Branch, Tehran, Iran.

His research interests include power control, wireless communications, array and

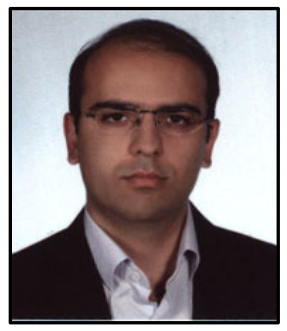
statistical signal processing, and smart antennas.

Hamidreza Bakhshi was born in Tehran, Iran on April 25, 1971. He received the B.Sc. degree in electrical engineering from Tehran University, Iran in 1992, the M.Sc. and Ph.D. degree in Electrical Engineering from Tarbiat Modarres University, Iran in 1995 and 2001, respectively. Since 2001, he has been as an Assistant Professor of Electrical Engineering at Shahed University, Tehran, Iran.

His research interests include wireless communications, multiuser detection, and smart antennas.

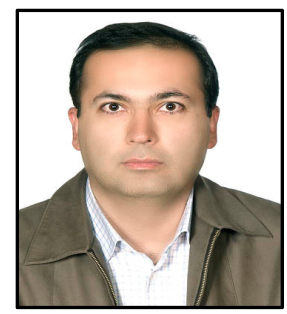

Gholamreza Dadashzadeh was born in Urmia, Iran, in 1964. He received the B.Sc. degree in communication engineering from Shiraz University, Shiraz, Iran in 1992 and M.Sc. and Ph.D. degree in communication engineering from Tarbiat Modarres University (TMU), Tehran, Iran, in 1996 and 2002, respectively. From 1998 to 2003, he has worked as head researcher of Smart Antenna for Mobile Communication Systems (SAMCS) and WLAN 802.11 project in radio communications group of Iran Telecomm Research Center (ITRC). From 2004 to 2008, he was dean of Communications Technology Institute (CTI) in ITRC. He is currently as Assistance Professor in the Department of Electrical Engineering at

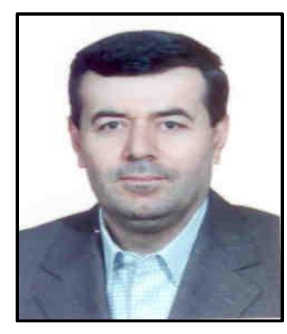
Shahed University, Tehran, Iran. He is a member of IEEE, Institute of Electronics, Information and Communication Engineers (IEICE) of Japan and Iranian Association of Electrical and Electronics Engineers (IAEEE) of Iran. He honored received the first degree of national researcher in 2007 from Iran's ministry of ICT.

His research interests include antenna design and smart antennas. 